\title{
Information gaps for patients requiring craniotomy for benign brain lesion: a qualitative study
}

\author{
Linda Rozmovits · Kathleen Joy Khu • \\ Soha Osman · Fred Gentili · Abhijit Guha • \\ Mark Bernstein
}

Received: 31 March 2009/Accepted: 22 June 2009/Published online: 3 July 2009

(C) The Author(s) 2009. This article is published with open access at Springerlink.com

\begin{abstract}
Doctor-patient communication in the setting of a life-threatening illness poses considerable challenges. This study aimed to determine the information needs of a subset of neurosurgical patients. Qualitative case study methodology was used. Twenty-five semi-structured interviews were conducted with ambulatory adult patients who had undergone surgery for a benign brain tumor, arteriovenous malformation, or unruptured aneurysm. Interviews were digitally audio recorded and transcribed, and the data subjected to thematic analysis. Six overarching themes emerged from the data: (1) the amount of information patients want varies; (2) the type of information needed is not limited to information about treatment options and risks; (3) patients engage in independent information seeking for a variety of reasons; (4) patients consider compassion from their surgeon as important; (5) direct communication with the surgeon post-operatively is very important; and (6) patients' information needs are greatest post-operatively. Many patients felt that the amount and quality of information they received was not sufficient, particularly regarding post-operative recovery and long-term life issues, leading many to do their own research. The findings from this study emphasize the need for improved communication with patients so they can participate meaningfully in choices about their treatment, give a truly informed consent, and effectively participate in their own recovery.
\end{abstract}

L. Rozmovits - K. J. Khu - S. Osman · F. Gentili · A. Guha ·

M. Bernstein $(\square)$

Division of Neurosurgery, Toronto Western Hospital, University of Toronto, 399 Bathurst Street, 4 West Wing, Toronto, ON M5T

2S8, Canada

e-mail: mark.bernstein@uhn.on.ca
Keywords Information needs · Neurosurgery · Qualitative research

\section{Introduction}

Being diagnosed with a life-threatening but benign neurosurgical condition such as a benign brain tumor is a major life event that brings with it complex and constantly evolving information and support needs. Given the risk of morbidity and/or mortality, not surprisingly the communication between doctors and their patients tends to focus on the immediate future with an emphasis on treatment options and risk. In the current climate of full disclosure, patients are often overwhelmed with statistical information that they are ill equipped to interpret and which may not address their real concerns. When such conversations take place between surgeons and patients who face possible morbidity after surgical intervention, it can be especially challenging to ensure that all the patient's information needs are met.

There are few studies [1-6] dealing with what patients want to know in the context of informed consent, but none address the information needs of patients undergoing major life-saving surgery. In addition, there has been little indepth work exploring the totality of patient experience from early signs and symptoms, through treatment, the post-operative period, and longer-term recovery. For patients, the lived experience of diagnosis, treatment, and recovery resonates well beyond the limited encounters they have with their surgeon. In order to map the currently uncharted territory of their information needs, a more detailed and comprehensive understanding of the patient journey is needed.

This study was conducted to explore the information needs of neurosurgical patients with non-malignant but 
potentially life-threatening conditions, and to provide evidence to support the development of more patientcentered approaches to doctor-patient communication by neurosurgeons.

\section{Methodology}

\section{Study design}

This was a qualitative research study using in-depth semistructured interviews with patients who had undergone craniotomy for a non-malignant but life-threatening intracranial lesion. Patients with malignant tumors were excluded as it was felt that their post-operative experience would be dominated by larger issues related to further adjuvant treatment and concerns about survival. Also, these patients have in-depth, regular, and consistent contact with health care providers for years after their surgery.

\section{Setting and participants}

Participants were patients recruited from the practices of five neurosurgeons specializing in brain tumors and cerebrovascular disease in a tertiary referral hospital. This is a teaching hospital with Acute Care Nurse Practitioners, residents, and fellows who take part in the patients' clinical management and also play some role in addressing patients' information needs. In addition, patients are provided with written pre- and post-operative information booklets addressing common concerns and questions.

The participants were ambulatory adult patients age 18 or older, proficient in English, who underwent surgery between April 2006 and February 2008. Recruitment of participants was led by a dedicated recruiter (SO) who attended the neurosurgical clinic for a 2-month-period. Patients were approached in a non-consecutive manner, attempting to provide a representative blend of patients vis a vis age, gender, specific benign diagnosis, and all five participating surgeons' practices. Prospective participants were identified and approached by the recruiter who explained the study and provided them with printed information that they could consider at their leisure. The information included a reply slip that they could return to indicate an interest in participating in the study. Those who returned the reply slips were subsequently contacted by an experienced qualitative researcher and interviewer (LR).

\section{Sample size}

Twenty-five interviews were sought as it was anticipated that this would be sufficient to achieve data saturation. "Saturation" is a concept in qualitative research describing the situation wherein no new concepts arise during successive interviews, beyond those that have already emerged [7].

Data collection

Twenty-five semi-structured interviews were conducted over a period of 2 months on patients who fulfilled the inclusion criteria. The semi-structured format following an interview guide (Appendix A) allows for both directed questions from the interviewer and freer exploration of unanticipated issues raised by the participants. All interviews were digitally audio recorded for verbatim transcription. Demographic data including age, sex, ethnicity, occupation, and diagnosis were collected.

\section{Data analysis}

Verbatim transcripts of all interviews were prepared by a professional transcriptionist, checked for accuracy against the sound files by the interviewer, and corrected where necessary. Transcripts were entered into HyperResearch software for qualitative data analysis and coded for both anticipated and emergent themes. A coding framework was developed in discussion with the principal investigator. For the analysis, the method of constant comparison was used and included searches for disconfirming evidence to ensure that all perspectives were represented. The process of constant comparison is part of a grounded theory approach to qualitative data analysis. Grounded theory is inductive, allowing analytical categories to emerge from the data "rather than defining them a priori." Constant comparison is a process by which data are compared with each other to define the integrity of these analytic categories $[7,8]$.

\section{Research ethics}

Participation was entirely voluntary and informed consent was obtained. All data were kept confidential. Audiotapes and anonymized transcripts were held in a secure location. The study was approved by the Research Ethics Board of the University Health Network.

\section{Results}

Patient information

Forty patients were approached, 37 expressed interest in participating, and 25 were eventually interviewed; most "missed interviews" resulted from logistic reasons. The interviews were conducted over a 2 month interval between February and April 2008. The length of time since 
Table 1 Demographic data of the study participants

\begin{tabular}{|c|c|c|}
\hline Age (years) & ( 16 of 25 were $40-60$ years old) & \\
\hline \multirow[t]{2}{*}{ Sex } & Male & 11 \\
\hline & Female & 14 \\
\hline \multirow[t]{6}{*}{ Region of origin } & Canada/US & 11 \\
\hline & Europe & 6 \\
\hline & Africa & 3 \\
\hline & South America & 2 \\
\hline & Asia & 2 \\
\hline & New Zealand & 1 \\
\hline \multirow[t]{8}{*}{ Occupation } & Health and social care & 4 \\
\hline & Financial/legal & 4 \\
\hline & Service industry & 4 \\
\hline & Education & 3 \\
\hline & Creative industry & 3 \\
\hline & Skilled labor & 2 \\
\hline & Engineering & 2 \\
\hline & Others & 3 \\
\hline \multirow[t]{5}{*}{ Diagnosis } & Meningioma & 10 \\
\hline & Vestibular schwannoma & 7 \\
\hline & Aneurysm & 4 \\
\hline & AVM & 3 \\
\hline & Colloid cyst & 1 \\
\hline
\end{tabular}

the patient's surgery ranged from 2 to 24 months in order to contribute to maximum variation sampling [9], which allows the researcher to document diversity and commonality of experience. This also allows for exploration of the evolution of information and support needs over time. Patients' demographic data are shown in Table 1.

\section{Thematic analysis}

Six overarching themes emerged from the analysis. These themes are described and illustrated by verbatim quotes from participant interviews.

\section{There is variation in the amount of information patients wanted prior to surgery}

Information needs prior to surgery varied among the participants and depended on a number of factors including age, personality, and educational, social, and ethnic background. Patients ranged between wanting to know everything to leaving the decision to the surgeon. The majority of patients expressed a need for more detailed and more voluminous information than had been provided, and many felt strongly that this was crucial to their decision making about treatment and capacity to cope after surgery.
I would like to know everything, the risks involved, whether it be $1 \%$ or whether it be $10 \% \ldots$ I need to know all the risks even if it is a minor risk.

A small subset of elderly female patients wanted minimal information and preferred to put themselves in the hands of their surgeons.

You the doctor, you know better than I am. Whatever has to be done, have it done.

\section{The information patients wanted prior to surgery is not limited to treatment options and surgical risks}

While all participants confirmed the conventional expectation that information about treatment options and surgical risk would be provided, many were concerned about other issues. These include the surgeon's background, reputation, and experience, an overview of the actual surgical procedure, and expectations for their recovery, both short and long term. The patients' need for information about their surgeon reflected the vulnerability they felt at having to entrust their future to another person with whom they had no previously established relationship of trust. Having that information was important, because feeling that they had made an active and informed choice provided the patients with a sense of control during a very vulnerable period.

It's scary...you really are in shock, you're so nervous...you don't really understand what's going on...you lose control....and for me, anyway...my little bit of control was making that choice so I needed to know that I'd made that decision wisely.

A number of patients expressed the need for information about how their surgery would be carried out. For example, they wanted to know exactly where the tumor was, where the surgeon would go in, the likely size and shape of the incision, what important nerves were in the vicinity, and how their skull would be reconstructed afterwards. They also had concerns about post-operative expectations.

...What are you going to do to my head? How are you going to take it apart and how are you going to put it back together? And after the surgery how am I going to feel and what's the time-line for those feelings?

When these concerns were adequately addressed many participants felt less distressed, less helpless and better able to cope with their situation. When they were not, the additional uncertainty added to their distress and became a focal point for anxiety. 


\section{Patients engage in independent information seeking for a variety of reasons}

Many participants sought out information about their condition, treatment options, and surgeons and hospital, by independent means. They did this through family members or friends who had informal access to other medical practitioners, through Internet-based research, or through nonprofit organizations or support groups. The reasons for seeking out additional information included: (1) insufficient or contradictory information from the surgeon and other consulting clinicians; (2) patient's feelings of responsibility to do their own research; (3) wanting reassurance or supporting information; (4) attempting to locate treatment facilities and a surgeon they could trust; (5) wanting to feel less passive and more empowered through increasing their own knowledge base; and (6) intellectual curiosity. Participants who had informal access to medical practitioners generally relied on them for decision-making support in choosing amongst treatment options and for confirmation of the professional reputation of their surgeon.

...we had a family friend who was a doctor so I asked her...who she would recommend to see, whether she thought that we should have the surgery as well and she said that Dr. [neurosurgeon] was...well-qualified, he had a lot of experience, he was...highly regarded in the doctor community...

Oh I went all over the 'net...

...I discovered there was a symposium on acoustic neuroma so both myself and my husband went to that...

\section{Patients want compassion from their surgeon}

For most of the patients, it was not enough that their neurosurgeon was capable and confident. They also sought signs of compassion reflected in the surgeon's communication style, willingness to answer questions, and nonverbal gestures such as sitting down with the patient. Signs of a compassionate surgeon provided reassurance that the surgeon would treat the patient humanely and could be trusted to make decisions that could have a profound impact on the rest of their lives. Having this kind of emotional or human connection with the surgeon helped patients come to terms with what they were facing. When this was deficient it often compounded existing distress.

You feel that your life is in their hands...you want some reassurance that they're going to do the best they can...

...I want to feel that my doctor cares about doing a good job.
5. Direct communication with the surgeon post-operatively is very important

One of the most widely shared and strongly expressed concerns of participants was over the lack of adequate postoperative communication with their surgeon about what had happened during their surgery. From a communication standpoint most described this as one of the most frustrating and disappointing aspects of their whole experience. The period immediately following surgery was, for many, characterized by a pronounced need for specific information about what they had just been through as well as for direct reassurance from the surgeon. This need was frequently unmet and left participants confused about what had happened to them, and lacking the closure they needed to have confidence in their recovery and regain a sense of control over their lives. This concern was most strongly expressed by the patients who developed post-operative complications or did not recover as well or as fast as they expected to.

...after the operation, the doctor just going outside the door and look at you and say "Good-bye," that's that, five minutes. They don't sit down there and talk to you after the operation.

They told me that my surgery was eight and a half hours. What did they do...in eight and a half hours? That's a full day's work!...I guess I just needed him to say, "You're going to be fine," you know, "this is what to expect... and ease my mind a little bit that way...I didn't get any of that, you know...

Some patients felt that the surgeon was concerned only with the surgical challenge and lost some interest in the patient after the surgery.

...I think that as soon as he was done sewing me back up that was it for me, you know. He had the obligatory come by, say hello, "Okay, he's doing okay," and walk away...

The patients who did feel satisfied with the communication they had with their surgeon after surgery confirmed its importance to them. They felt that a direct communication with their surgeon was more meaningful than if the assistant talked with them or if the surgeon spoke with the patient's family and not the patient him/herself.

...I think it's very important because if the doctor doesn't show up and talk to you it might make you a little nervous. It's better to hear from them whether it's good or bad than hear nothing at all.

...I think the conversation from the doctor was very important...it just shows that the doctor cares and he just wants to make sure that you are comfortable in the sense that everything is okay now. 


\section{Patients' information needs are greatest} post-operatively

The majority of the patients remarked that their greatest information need arose after surgery. They felt that the information about recovery they were provided was often inadequate. For the patients who had a swift and uneventful recovery, there were still questions regarding everyday life and day-to-day issues which they did not have the answers to. For those who developed complications or unexpected symptoms, the information gap added considerably to their distress and compounded their anxiety. They did not know if what they were experiencing was expected or not, and whether they should be alarmed. While most patients had been informed of the risks of surgery, many experienced other symptoms that they did not expect and were not mentioned by the surgeon, such as fatigue, psychological disturbance, insomnia, and slow recovery.

...Nothing. I had no idea...Didn't know how long I'd be in the hospital, didn't know what condition I would be in once I got home, or while I was in the hospital...I had no idea.

The most consistent point of miscommunication was the length of time patients should allow for recovery, leading to a great deal of confusion and subsequent distress. Commonly, participants were told that it would be a matter of weeks, but later discovered that their postsurgical problems would persist for months or longer. While the provision of the typical "6-8 weeks" guideline may have referred to a particular set of concerns relevant to surgeons, it did not take into account the full range of physical, cognitive, psychological and emotional problems by which participants were affected and which had a substantial impact on both their physical and social capacity over the mid to long term.

He basically said that it will take...six to eight weeks...when I went to the six-week control...I said to him... "I'm really stiff" and he said, "Yeah, well, you know, you're going to have the numbness in your head because of the nerves regenerating..." and I said "So how long is this going to take?" and he says, "Oh six months to a year." And that was the first time I heard the six months to a year timeline...

Another source of distress was the patients' feeling of being abandoned and left on their own during recovery, when they were experiencing symptoms and didn't know where to turn.

...I felt that each doctor is concerned with doing an operation and having a success and putting a mark saying, "Hey, I have another successful operation.
You're on your own now. We did our job, now if there's any complications due to this thing here... that's not our problem.

\section{Discussion}

Although qualitative research is unfamiliar to most quantitatively oriented physicians, it is a powerful tool for gaining insight into patient perspectives and answering questions that cannot be answered by quantitative research methodology [10]. Qualitative findings are not generalizable; rather, they are indicative of a range of common experiences.

It is widely accepted that adequate information before a therapeutic procedure is fundamental to giving informed consent, and there is literature attesting to this [1-5, 11, 12]. However, information needs vary from patient to patient, and for many, the amount and type of information they seek exceeds that required to secure informed consent.

Some postulate that providing information about risks and complications causes undue and unnecessary anxiety, whereas others report that improving patient's knowledge about treatment reduces anxiety [11]. Beresford et al. [13] identified three groups of patients in terms of their information needs: (1) those requiring little or no risk information; (2) those requiring information about major risks; and (3) those requiring full risk disclosure. Because of this heterogeneity, patient preferences should be discussed before risk disclosure. Similarly, Bridson et al. [14] advocate a more patient-centered method in which the clinician should ask patients their goals for treatment even before treatment options are discussed, in order to tailor the information to fit the context of the patient's objectives. Fraser [15] suggests that doctors should neither insist on gaining fully informed consent from anxious patients nor deny detailed information to inquiring ones.

A few studies have been conducted to assess the effect of demographic factors on patient preference, and it was shown that younger individuals with a higher level of education require more information $[6,12]$. In our study subgroup analysis was not possible due to the small numbers in each group.

In terms of the amount of pre-operative information, most of the participants felt that what they received was sufficient to make a decision regarding surgery, but was lacking in other aspects of their illness. Other studies report a wide range in patient satisfaction with the amount of information they received, with the number of satisfied respondents ranging from $48 \%$ to $96 \%$ [1, 3, 11]. Rankinen et al. [6] conclude that the amount of information surgical patients receive is less than they expected. 
What do patients want to know? The list includes information about surgical risks [1-5], the nature of the disease and indications for surgery [3], surgical technique [2, 3], information about the surgeon [2], alternative treatment options [2, 4, 5], outcome, and quality and quantity of life [5]. Aside from information that would help them decide whether or not they will accept surgery, patients also value information about what to expect post-operatively. Specifically, information about recovery time, future management, and long-term effect on work are highly desired [2-4]. This is consistent with the finding in this study that patients' information needs are greater post-operatively. This can be explained by an increased demand on the part of the patient and a decreased supply on the part of the surgeon. Preoperatively, thoughts about the surgery and its possible complications occupy the patient's mind; it is only after the surgery is over that they allow themselves to think about the recovery period and long term sequelae of their illness. Also, surgeons generally focus on the large task, not the "smaller" issues that are of concern to patients.

There is also an understandable tendency for surgeons to focus on the treatment itself and the events preceding it rather than those following it, resulting in a mismatch in information needs and delivery for patients. This results in significant information deficits around treatment outcomes, expectations of recovery, and the return to health, with some participants feeling that they were simply cut adrift as soon as they were "off the table." Ironically, patients with more serious diagnoses, like malignancy, are probably less likely to experience what patients with benign tumors do, likely because of the intensity and frequency of monitoring, doctor contact, and ongoing communication in this group of patients.

Inadequate post-operative information has been observed in other settings. Henderson and Phillips [16] reported that only $55 \%$ of patients routinely received discharge information, and the $45 \%$ had to ask for information if they wanted it. Information about their illness and recovery helps people cope better, enabling them to comply with the post-treatment constraints and to recognize and act appropriately should there be any complications [3]. In addition, pre-operative knowledge reduces anxiety, pain, and stress, the need for physiotherapy and occupational therapy, and the length of hospital stay [6].

Could a long interval between surgery and the interview introduce "recall bias"? Since qualitative interviews are not tests of recall, the concept of recall bias is not relevant. Qualitative research seeks to address questions that cannot be answered by counting or measuring things or, indeed, testing them against objective markers. What people remember and how they remember it provides important and useful information about how they experienced illness and healthcare. While narrative accounts of experience are undoubtedly shaped and re-shaped over time, this is seen as adding depth and insight rather than detracting from the value of the information.

The need for information begins when the illness starts [12], which does not necessarily correspond to when the patient meets the surgeon. As a result, several patients take it upon themselves to self-advocate and do their own research. Others do the same even after they have spoken with the surgeon. While this behavior is a feature of the information age in which we live, it is also an expression of the vulnerability people feel at having to rely on health care providers for information and the low expectations they have of their information needs being met.

Regarding the use of the internet, some patients access credible resources such as the website of the treating hospital and organizations such as the acoustic neuroma association, while others get their information from less reliable sources such as patients' blogs and online patient chat rooms. Several participants who found themselves alarmed at information they felt unable to assess adequately stopped themselves from looking any further for this reason.

The findings from this study emphasize the need for improved communication with all patients, so they can effectively participate in their recovery process and move on with their lives. Many of these information needs can be met by the involvement of allied health care providers (e.g. nurses, social workers), and thorough but easy to read written materials. These adjuncts should be optimally used to help busy practitioners better serve their patients. However, neurosurgeons (along with some help from other allied health care personnel) must carry the responsibility to avoid information gaps and optimize communication with their patients.

Acknowledgment The authors thank Chris Wallace, MD, MSc, FRCSC, FACS and Michael Tymianski, MD, PhD, FRCSC at the Toronto Western Hospital for allowing us to study their patients, and to all the patients for their generosity and grace. We also acknowledge two grants: (1) Canadian Institutes of Health Research, MOP 77670, Therapeutic Hopes and Ethical Concerns: Clinical Research in the Neurosciences; and (2) Canadian Institutes of Health Research, NNF 80045, States of Mind: Emerging Issues in Neuroethics for help supporting this work.

Open Access This article is distributed under the terms of the Creative Commons Attribution Noncommercial License which permits any noncommercial use, distribution, and reproduction in any medium, provided the original author(s) and source are credited.

\section{Appendix A: interview guide}

Preamble: We are trying to learn more about what neurosurgical patients need and want to know before they undergo surgery so that we can improve the communication that takes place between doctors and their patients. 
1. How did you find out that you had a brain tumor? (prompt: When did you first suspect something might be wrong-when were you first told that it was a brain tumor? Can you describe the conversation for me?)

2. What was your reaction to the news? (prompt: Were you frightened, panicked, or relieved to know what was wrong with you? Did you fear you were going to die?)

3. Did you know anything about brain tumors or neurosurgery before this happened to you?

(prompt: Do you know anyone else who had a similar experience? Had you seen anything on TV or in the press? Did you have an image in your mind of what it might be like to have one?)

4. Tell me more about the conversation you had with your surgeon prior to the surgery.

(prompt: How were your choices presented to you? What did he tell you about the risks involved? Did he provide you with statistical information about risk? Were you able to understand and take in everything that he was saying?)

5. How did you feel when you left the room after that conversation?

(prompt: Did you have a better understanding of your situation? Were you feeling overwhelmed by the information? Did you feel clearer about some things but still confused about others? What were they?)

6. When you went home did you talk over the information you'd been given with anyone?

(prompt: Was it easy to remember what you had been told? Did you forget some of what you'd been told? In talking things over did you discover things you'd forgotten to ask or still felt confused about?)

7. After your conversation with the surgeon, did you seek out any more information about your condition on your own?

(prompt: Did you look things up on the internet or ask someone to do this for you? Did you call a support organization, look things up in a medical book, ask someone you knew? What were you trying to find out?)

8. Knowing what you know now, how do you feel about the way information was presented to you before surgery?

(prompt: Did it provide you with the information you needed? Was it too technical? Was there not enough, too much, the right amount? Was the timing of the conversation appropriate? Would you have liked written information to take away?)

9. What advice would you offer surgeons about how to talk to people in your situation? (prompt: What kind of information should they provide? How should they provide it? What about the timing of the conversation?)

10. What advice would you offer other patients in your situation in order to ensure that they find out everything they need to know?

11. Is there anything else about your information needs in relation to your surgery that we haven't talked about that was important to you?

\section{References}

1. Adhikari P, Pradhananga RB (2007) Patients' expectations on informed consent before ENT surgery. Int Arch Otolaryngol $11: 1-5$

2. Courtney MJ (2001) Information about surgery: what does the public want to know? ANZ J Surg 71:24-26

3. Dawes PJ, Davison P (1994) Informed consent: what do patients want to know? J R Soc Med 87:149-152

4. El-Wakeel H, Taylor GJ, Tate JJT (2006) What do patients really want to know in an informed consent procedure? A questionnaire-based survey of patients in the Bath area. UK. J Med Ethics 32:612-616

5. Newton-Howes PA, Bedford ND, Dobbs BR, Frizelle FA (1998) Informed consent: what do patients want to know? N Z Med J 111:340-342

6. Rankinen S, Salantera S, Heikkinen K, Johansson K, Kaljonen A, Virtanen H, Leino-Kilpi H (2007) Expectations and received knowledge by surgical patients. Int J Qual Health Care 19: 113-119

7. Strauss A, Corbin J (1998) Basics of qualitative research: techniques and procedures for developing grounded theory. Thousand Oaks, CA, Sage Publications, pp 55-242

8. Pope C, Mays N, Ziebland S (2000) Analysing qualitative data. In: Pope C, Mays N (eds) Qualitative research in health care. BMJ Books, London, p 78

9. Miles M, Huberman AM (1994) Qualitative data analysis. Thousand Oaks CA, Sage Publications, pp 28-29

10. Krupp W, Klein C, Koschny R, Holland H, Seifert V, Meixensberger J (2009) Assessment of neuropsychological parameters and quality of life to evaluate outcome in patients with surgically treated supratentorial meningiomas. Neurosurgery 64:40-47

11. Ghulam AT, Kessler M, Bachmann LM, Haller U, Kessler TM (2006) Patients' satisfaction with the preoperative informed consent procedure: a multicenter questionnaire survey in Switzerland. Mayo Clin Proc 81:307-312

12. Ivarsson B, Larsson S, Luhrs C, Sjoberg T (2005) Extended written pre-operative information about possible complications at cardiac surgery-do the patients want to know. Eur J Cardiothorac Surg 28:407-414

13. Beresford N, Seymour L, Vincent C, Moat N (2001) Risks of elective cardiac surgery: what do patients want to know? Heart 86:626-631

14. Bridson J, Hammond C, Leach A, Chester M (2003) Making consent patient centred. Br Med J 327:1159-1161

15. Fraser AG (1984) Do patients want to be informed? A study of consent for cardiac catheterisation. Br Heart J 52:468-470

16. Henderson A, Phillips S (1996) Surgical patients' information needs on discharge: are they being met? Int $\mathrm{J}$ Nurs Pract 2: 229-235 